\title{
Three planetary companions around M 67 stars $^{\star}, \star \star$
}

\author{
A. Brucalassi ${ }^{1,2}$, L. Pasquini ${ }^{3}$, R. Saglia ${ }^{1,2}$, M. T. Ruiz ${ }^{4}$, P. Bonifacio ${ }^{5}$, L. R. Bedin ${ }^{6}$, K. Biazzo $^{7}$, C. Melo ${ }^{8}$, \\ C. Lovis ${ }^{9}$, and S. Randich ${ }^{10}$ \\ 1 Max-Planck Institut für extraterrestrische Physik, 85740 Garching bei München, Germany \\ e-mail: abrucala@mpe.mpg.de \\ 2 University Observatory Munich, Ludwig Maximillian Universitaet, Scheinerstrasse 1, 81679 Munich, Germany \\ 3 ESO - European Southern Observatory, Karl-Schwarzschild-Strasse 2, 85748 Garching bei München, Germany \\ 4 Astronomy Department, Universidad de Chile, 36-D Casilla, Santiago, Chile \\ 5 GEPI, Observatoire de Paris, CNRS, Univ. Paris Diderot, Place Jules Janssen, 92190 Meudon, France \\ ${ }^{6}$ Istituto Nazionale di Astrofisica, Osservatorio Astronomico di Padova, 35122 Padova, Italy \\ 7 Istituto Nazionale di Astrofisica, Osservatorio Astronomico di Catania, 95123 Catania, Italy \\ ${ }^{8}$ ESO - European Southern Observatory, 19001 Santiago, Chile \\ 9 Observatoire de Genève, 1290 Sauverny, Switzerland \\ 10 Istituto Nazionale di Astrofisica, Osservatorio Astrofisico di Arcetri, Firenze, Italy \\ Received 1 September 2013 / Accepted 13 December 2013
}

\section{ABSTRACT}

For the past six years we have carried out a search for massive planets around main sequence and evolved stars in the open cluster M 67, using radial velocity (RV) measurements obtained with HARPS at ESO (La Silla), SOPHIE at OHP and HRS at HET. Additional $\mathrm{RV}$ data come from CORALIE at the Euler Swiss Telescope. We aim to perform a long-term study on giant planet formation in open clusters and determine how it depends on stellar mass and chemical composition. We report the detection of three new extrasolar planets: two in orbit around the two G dwarfs YBP1194 and YBP1514, and one around the evolved star S364. The orbital solution for YBP1194 yields a period of 6.9 days, an eccentricity of 0.24 , and a minimum mass of $0.34 M_{\text {Jup }}$ YBP1514 shows periodic RV variations of 5.1 days, a minimum mass of $0.40 M_{\mathrm{Jup}}$, and an eccentricity of 0.39 . The best Keplerian solution for S364 yields a period of 121.7 days, an eccentricity of 0.35 and a minimum mass of $1.54 M_{\text {Jup }}$. An analysis of $\mathrm{H} \alpha$ core flux measurements as well as of the line bisectors spans revealed no correlation with the RV periods, indicating that the RV variations are best explained by the presence of a planetary companion. Remarkably, YBP1194 is one of the best solar twins identified so far, and YBP1194b is the first planet found around a solar twin that belongs to a stellar cluster. In contrast with early reports and in agreement with recent findings, our results show that massive planets around stars of open clusters are as frequent as those around field stars.

Key words. planets and satellites: detection - planetary systems - open clusters and associations: individual: M 67

\section{Introduction}

In 2008 we began monitoring the radial velocities (RVs) of a sample of main sequence and giant stars in the open cluster (OC) M 67, to detect signatures of giant planets around their parent stars. An overview of the sample and of our first results is reported in Pasquini et al. (2012). The goal of this campaign is to study the formation of giant planets in OCs to understand whether a different environment, such as a rich cluster like M 67, might affect the planet formation process, the frequency, and the evolution of planetary systems with respect to field stars. In addition, searching for planets in OCs enables us to study the dependence of planet formation on stellar mass and to compare the chemical composition of stars with and without planets in detail. Stars in OCs share age and chemical composition (Randich et al. 2005), therefore it is possible to strictly control the sample and to limit the space of parameters in a better way than when studying field stars. To address these questions we started a search for planets around stars of the OC M67. This cluster

* Based on observations collected at the ESO $3.6 \mathrm{~m}$ telescope (La Silla), at the $1.93 \mathrm{~m}$ telescope of the Observatoire de HauteProvence (OHP) and at the Hobby Eberly Telescope (HET).

$\star \star$ Appendix $\mathrm{A}$ is available in electronic form at http://www . aanda.org has solar age (3.5-4.8 Gyr; Yadav et al. 2008) and solar metallicity (+0.03 $\pm 0.01 \mathrm{dex}$; Randich et al. 2006). In this Letter, we present the RV data obtained for the stars YBP1194, YBP1514, and S364 that reveal the presence of Jovian-mass companions.

\section{Stellar characteristics}

The three stars belong to the M 67 sample presented in Pasquini et al. (2012) with a proper motion membership probability higher than $60 \%$ according to Yadav et al. (2008) and Sanders (1977), see Appendix A. The basic stellar parameters $\left(V, B-V, T_{\text {eff }}, \log g\right.$ and $\left.[\mathrm{Fe} / \mathrm{H}]\right)$ with their uncertainties were adopted from the literature. Considering a distance modulus of $9.63 \pm 0.05$ (Pasquini et al. 2008) and a reddening of $E(B-V)=0.041 \pm 0.004$ (Taylor 2007), stellar masses and radii were estimated using the $4 \mathrm{Gyr}$ theoretical isochrones from Pietrinferni et al. (2004) and Girardi et al. (2000). The parameters derived from isochrone fitting are comparable, within the errors, with the values adopted from the literature. The main characteristics of the three host stars are listed in Table 1 . We note that the errors on these values do not include all potential systematics (see Appendix A).

YBP1194 is a G5V star, described by Pasquini et al. (2008) as one of the five best solar analogs in their sample. A detailed 
Table 1. Stellar parameters of the three M 67 stars hosting planets.

\begin{tabular}{lrrr}
\hline \hline Parameters & YBP1194 & YBP1514 & SAND364 \\
\hline$\alpha(\mathrm{J} 2000)$ & $08: 51: 00.81$ & $08: 51: 00.77$ & $08: 49: 56.82$ \\
$\delta(\mathrm{J} 2000)$ & $+11: 48: 52.76$ & $+11: 53: 11.51$ & $+11: 41: 33.00$ \\
Spec. type & $\mathrm{G} 5 \mathrm{~V}$ & $\mathrm{G} 5 \mathrm{~V}$ & $\mathrm{~K} 3 \mathrm{III}$ \\
$m_{\mathrm{V}}[\mathrm{mag}]$ & $14.6^{a}$ & $14.77^{a}$ & $9.8^{b}$ \\
$B-V[\mathrm{mag}]$ & $0.626^{a}$ & $0.680^{a}$ & $1.360^{b}$ \\
$M \star\left[M_{\odot}\right]$ & $1.01 \pm 0.02^{c}$ & $0.96 \pm 0.01^{d}$ & $1.35 \pm 0.05^{d}$ \\
$R \star\left[R_{\odot}\right]$ & $0.99 \pm 0.02^{d}$ & $0.89 \pm 0.02^{d}$ & $21.8 \pm 0.7^{d}$ \\
$\log g[\mathrm{cgs}]$ & $4.44 \pm 0.035^{c}$ & $4.57 \pm 0.05^{e}$ & $2.20 \pm 0.06^{f}$ \\
$T_{\mathrm{eff}}[\mathrm{K}]$ & $5780 \pm 27^{c}$ & $5725 \pm 45^{e}$ & $4284 \pm 9^{f}$ \\
{$[\mathrm{Fe} / \mathrm{H}][\mathrm{dex}]$} & $0.023 \pm 0.015^{c}$ & $0.03 \pm 0.05^{e}$ & $-0.02 \pm 0.04^{f}$ \\
\hline
\end{tabular}

References. ${ }^{(a)}$ Yadav et al. (2008). ${ }^{(b)}$ Montgomery et al. (1993). (c) Önehag et al. (2011). ${ }^{(d)}$ Pietrinferni et al. (2004) and Girardi et al. (2000). ${ }^{(e)}$ Smolinski et al. (2011) and Lee et al. (2008). ${ }^{(f)} \mathrm{Wu}$ et al. (2011).

spectroscopic analysis (Önehag et al. 2011) has confirmed the star as one of the best-known solar-twins.

YBP1514 also is a G5 main sequence star. We adopted the atmospheric parameters obtained by Smolinski et al. (2011), who used spectroscopic and photometric data from the original Sloan Digital Sky Survey (SDSS-I) and its first extension (SDSS-II/SEGUE). These values are consistent, within the errors, with what has been found in previous work on the same data by Lee et al. (2008) and in the study of Pasquini et al. (2008).

S364 (MMJ6470) is an evolved K3 giant star. The stellar parameters, summarized in Table 1, are taken from $\mathrm{Wu}$ et al. (2011). We derived its mass and radius by isochrone fitting (Pietrinferni et al. 2004).

\section{Radial velocities and orbital solutions}

The RV measurements were obtained using the HARPS spectrograph (Mayor et al. 2003) at the ESO $3.6 \mathrm{~m}$ telescope in high-efficiency mode (with $R=90000$ and a spectral range of 378-691 nm); the SOPHIE spectrograph (Bouchy $\&$ SOPHIE Team 2006) at the OHP $1.93 \mathrm{~m}$ telescope in high-efficiency mode (with $R=40000$ and a spectral range of 387-694 nm), and the HRS spectrograph (Tull 1998) at the Hobby Eberly Telescope (with $R=60000$ and a wavelength range of 407.6-787.5 $\mathrm{nm}$ ). In addition, we gathered RV data points for giant stars observed between 2003 and 2005 (Lovis \& Mayor 2007) with the CORALIE spectrograph at the $1.2 \mathrm{~m}$ Euler Swiss telescope. HARPS and SOPHIE are provided with a similar automatic pipeline to extract the spectra from the detector images and to cross-correlate them with a G2-type mask obtained from the Sun spectra. Radial velocities are derived by fitting each resulting cross-correlation function (CCF) with a Gaussian (Baranne et al. 1996; Pepe et al. 2002). For the HRS, the RVs were computed using a series of dedicated routines based on IRAF and cross-correlating the spectra with a G2 star template (Cappetta et al. 2012). All the observations for each star were corrected to the zero point of HARPS, as explained in Pasquini et al. (2012), and were analyzed together. Two additional corrections were applied to the SOPHIE data, to take into account the modification of the fiber link in June 2011 (Perruchot et al. 2011) and the low $\mathrm{S} / \mathrm{N}$ ratio of the observations. For the first, we calculated the offset between RV values of our stellar standard (HD 32923) before and after the change of the optical setup. For the second, we corrected our spectra using Eq. (1) in Santerne et al. (2012). We studied the $\mathrm{RV}$ variations of our target stars by computing the Lomb-Scargle
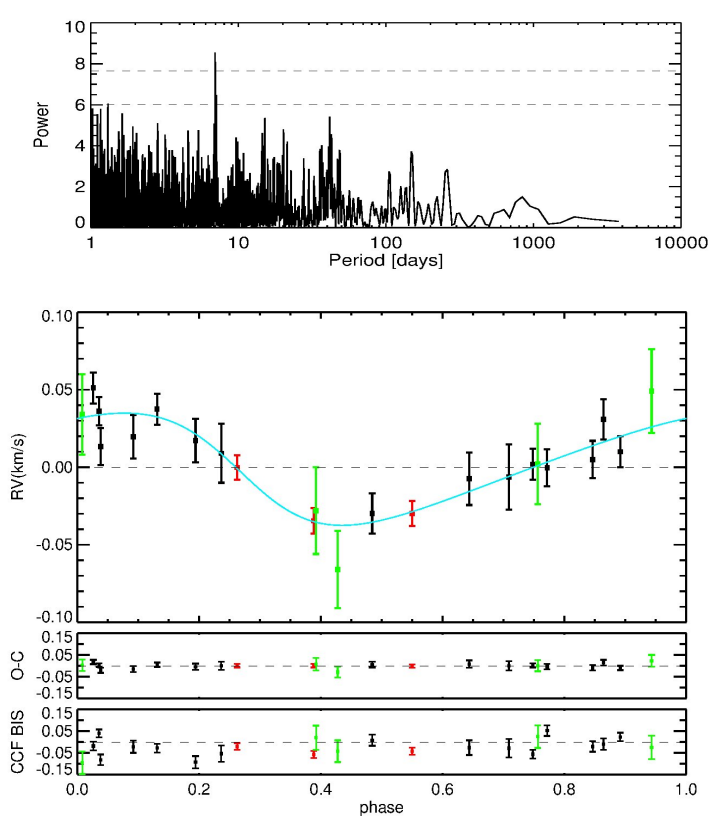

Fig. 1. Top: Lomb-Scargle periodogram for YBP1194. The dashed lines correspond to $5 \%$ and $1 \%$ false-alarm probabilities, calculated according to Horne \& Baliunas (1986) and white noise simulations. Bottom: phased RV measurements and Keplerian best fit, best-fit residuals, and bisector variation for YBP1194. Black dots: HARPS measurements, red dots: SOPHIE measurements, green dots: HRS measurements.

periodogram (Scargle 1982; Horne \& Baliunas 1986) and by using a Levenberg-Marquardt analysis (Wright \& Howard 2009, RVLIN) to fit Keplerian orbits to the RV data. The orbital solutions were independently checked using the Yorbit program (Segransan et al., in prep.). For each case we verified that the RVs did not correlate with the bisector span of the CCF (calculated following Queloz et al. 2001) or with the FWHM of the CCF. All the RV data for each star are available in Appendix A.

\section{YBP1194}

We have acquired 23 RV measurements since 2008. Fifteen were obtained with HARPS with a typical $\mathrm{S} / \mathrm{N}$ of 10 (per pixel at 550 $\mathrm{nm}$ ), leading to a mean measurement uncertainty of $13 \mathrm{~m} \mathrm{~s}^{-1}$ including calibration errors. Eight additional RV measurements were obtained with SOPHIE and HRS with mean measurement uncertainties of $9.0 \mathrm{~m} \mathrm{~s}^{-1}$ and $26.0 \mathrm{~m} \mathrm{~s}^{-1}$. A clear 6.9 day periodic signal can be seen in the periodogram (see Fig. 1 top) with its one-year and two-year aliases on both sides (at $6.7 \mathrm{~d}$ and $7.03 \mathrm{~d}$ ). A single-Keplerian model was adjusted to the data (Fig. 1 bottom). The resulting orbital parameters for the planet candidate are reported in Table 2. The residuals' dispersion is $\sigma(\mathrm{O}-\mathrm{C})=11.55 \mathrm{~m} \mathrm{~s}^{-1}$, comparable with the mean measurement accuracy $\left(\sim 15 \mathrm{~m} \mathrm{~s}^{-1}\right)$, and the periodogram of the residuals does not show significant power excess, although structures are present.

\section{YBP 1514}

Twenty-five RV measurements have been obtained for YBP1514 since 2009: 19 with HARPS, the others with HRS and SOPHIE. The typical $\mathrm{S} / \mathrm{N}$ is $\sim 10$ and the measurement uncertainty is $\sim 15 \mathrm{~m} \mathrm{~s}^{-1}$ for HARPS, $\sim 25 \mathrm{~m} \mathrm{~s}^{-1}$ for HRS, and $\sim 10 \mathrm{~m} \mathrm{~s}^{-1}$ for SOPHIE. A significant peak is present in the periodogram at 5.11 days (Fig. 2 top), together with its one-year alias at 5.04 days. We fitted a single-planet Keplerian orbit corresponding to the period $P=5.11$ days (Fig. 2 bottom). The orbital parameters resulting from this fit are listed in Table 2. Assuming a mass of $0.96 M_{\odot}$ for the host star, we computed a minimum 
Table 2. Orbital parameters of the planetary companions.

\begin{tabular}{lrrr}
\hline \hline Parameters & YBP1194 & YBP1514 & SAND364 \\
\hline$P$ [days $]$ & $6.958 \pm 0.001$ & $5.118 \pm 0.001$ & $121.710 \pm 0.305$ \\
$T[\mathrm{JD}]$ & $2455978.8 \pm 0.5$ & $2455986.3 \pm 0.3$ & $2456240.9 \pm 3.7$ \\
$e$ & $0.24 \pm 0.08$ & $0.39 \pm 0.17$ & $0.35 \pm 0.08$ \\
$\omega[\mathrm{deg}]$ & $98.62 \pm 25.68$ & $327.49 \pm 16.05$ & $273.51 \pm 12.81$ \\
$K\left[\mathrm{~m} \mathrm{~s}^{-1}\right]$ & $37.72 \pm 4.27$ & $52.29 \pm 10.39$ & $67.42 \pm 5.85$ \\
$m \sin i\left[M_{\text {Jup }}\right]$ & $0.34 \pm 0.05$ & $0.40 \pm 0.11$ & $1.54 \pm 0.24$ \\
$\gamma\left[\mathrm{km} \mathrm{s}^{-1}\right]$ & $34.184 \pm 0.006$ & $34.057 \pm 0.017$ & $33.217 \pm 0.018$ \\
$\sigma(\mathrm{O}-\mathrm{C})\left[\mathrm{m} \mathrm{s}^{-1}\right]$ & 11.55 & 14.6 & 15.0 \\
\hline
\end{tabular}

Notes. $P$ : period, $T$ : time at periastron passage, $e$ : eccentricity, $\omega$ : argument of periastron, $K$ : semi-amplitude of RV curve, $m \sin i$ : planetary minimum mass, $\gamma$ : average radial velocity, $\sigma(\mathrm{O}-\mathrm{C})$ : dispersion of Keplerian fit residuals.
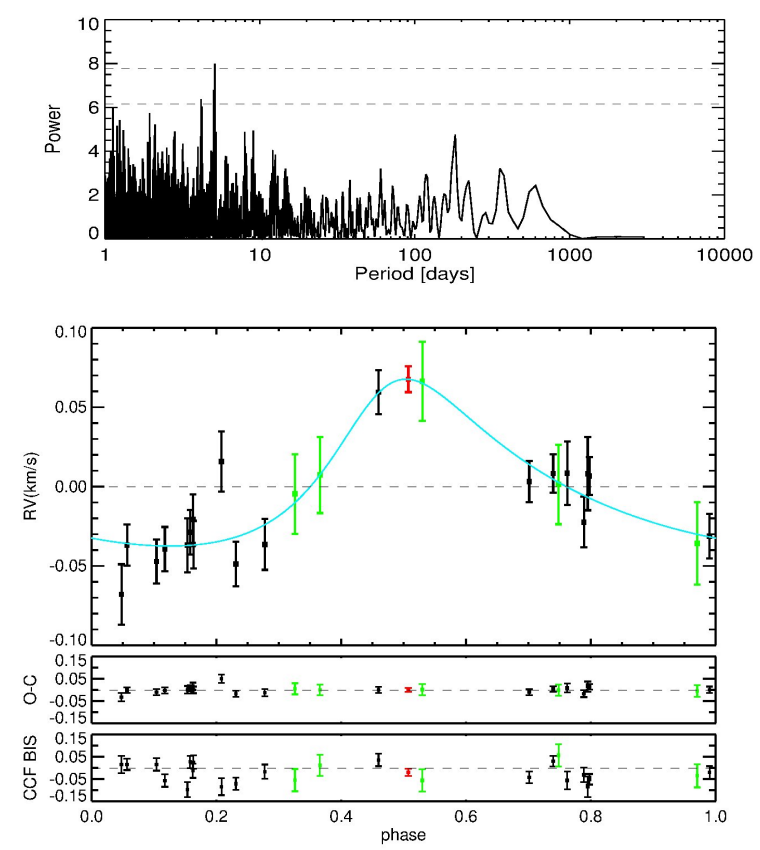

Fig. 2. Top: Lomb-Scargle periodogram for YBP1514. Bottom: phased RV measurements and Keplerian best fit, best-fit residuals, and bisector variation for YBP1514. Same symbols as in Fig. 1.

mass for the companion of $0.40 \pm 0.11 M_{\text {Jup }}$. The residuals to the fitted orbit have a dispersion of $\sigma(\mathrm{O}-\mathrm{C})=14.6 \mathrm{~m} \mathrm{~s}^{-1}$, within the mean measurement uncertainty, and show no significant periodicity.

\section{S364}

We collected $20 \mathrm{RV}$ measurements of S364 in about four years with HARPS, HRS, and SOPHIE. The average RV uncertainty is $\sim 3.0 \mathrm{~m} \mathrm{~s}^{-1}$ for HARPS, $\sim 7.0 \mathrm{~m} \mathrm{~s}^{-1}$ for SOPHIE and $\sim 20 \mathrm{~m} \mathrm{~s}^{-1}$ for HRS. Seven additional RV measurements were obtained with CORALIE between 2003 and 2005, with a mean measurement uncertainty of $\sim 12 \mathrm{~m} \mathrm{~s}^{-1}$. The periodogram of the observed data is shown in Fig. 3 (top) and indicates an excess of power at $\approx 121.7$ days. The other clearly visible peak at 182 days is the one-year alias of the planetary signal at $P=121.7$ days. It disappears in the periodogram of residuals, which no longer shows any signal. We fitted a single-planet Keplerian orbit to this signal (Fig. 3 bottom) and found an orbital solution whose parameters are reported in Table 2. The residuals to the fitted orbit show a level of variation of $\sigma=16.0 \mathrm{~m} \mathrm{~s}^{-1}$, higher than the estimated accuracy, but the periodogram of the residuals does not reveal significant peaks.
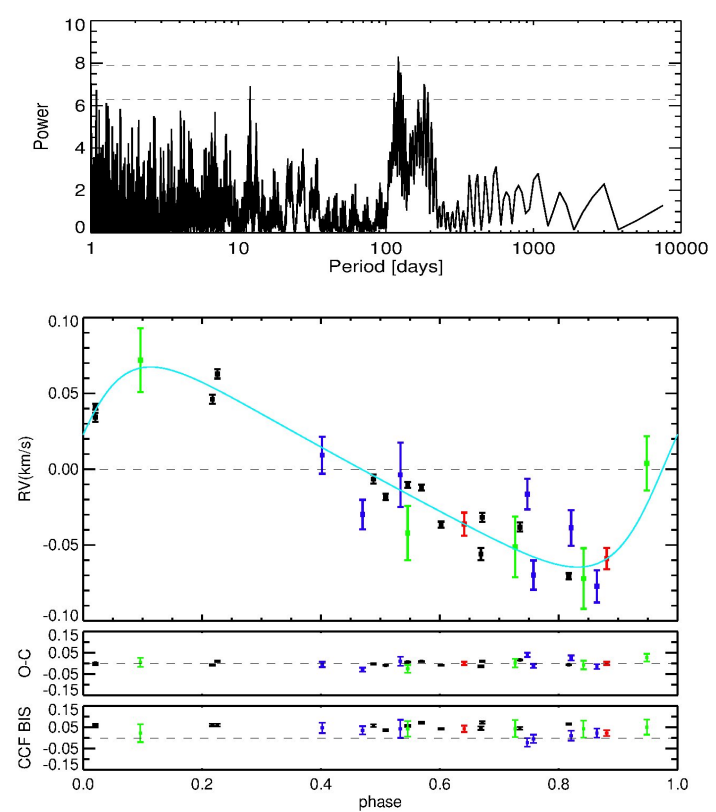

Fig. 3. Top: Lomb-Scargle periodogram for S364. Bottom: phased RV measurements and Keplerian best fit, best-fit residuals, and bisector variation for S364. Same symbols as in Fig. 1.

\section{Discussion and prospects}

We have presented new results from our planet-search campaign in the OC M67. Our measurements reveal that Y1194, Y1514, and S364 host planets.

To rule out activity-related rotational modulation as the cause of the RV variations in our object data, we investigated chromospheric activity in these stars by measuring the variations of the core of $\mathrm{H} \alpha$ with respect to the continuum. The low $\mathrm{S} / \mathrm{N}$ ratio of our observations does not provide sufficient signal in the region of the more sensitive $\mathrm{Ca} \mathrm{II} \mathrm{H}$ and $\mathrm{K}$ lines. We followed a method similar to the one described in Pasquini \& Pallavicini (1991). All the targets exhibit a very low level of activity: S364 shows a variability in $\mathrm{H} \alpha$ of 2\%, YBP1514 and YBP1194 of 3\% without significant periodicity. In addition, the M 67 stars have a very low level of chromospheric activity (Pace \& Pasquini (2004): $\left\langle F_{K}^{\prime}\right\rangle \sim 0.5 \times 10^{6} \mathrm{erg} \mathrm{cm}^{-2} \mathrm{~s}^{-1}$ for M 67 compared with $\left\langle F_{K}^{\prime}\right\rangle \sim 2.1 \times 10^{6} \mathrm{erg} \mathrm{cm}^{-2} \mathrm{~s}^{-1}$ for the Hyades), which is not compatible with generating the high RV variations we observe. Therefore, rotationally modulated RV variations for the dwarfs in M 67 are certainly not a concern. The remote possibility that these stars are short-period binaries seen pole-on can also be excluded, because they are very active, and will show enhanced $\mathrm{H} \alpha$ cores and strong X-ray emission, which has not been observed for these stars (van den Berg et al. 2004). The fact that these stars are of solar age and that our research is focused on finding giant planets with an expected RV variability of tens of $\mathrm{m} \mathrm{s}^{-1}$ makes the contamination by activity irrelevant.

It is remarkable that Y1194 is one of the best-known solar twins. This star together with Y1514, S364, and the other M 67 targets will be suitable for a detailed differential abundance analysis to compare the chemical composition of stars with and without giant planets.

All the orbital solutions show nonzero eccentricity, but this is also common among planets found around field stars. Quinn et al. (2013) explained that hot-Jupiters in OCs with nonzero eccentric orbits and circularization time-scales $t_{\text {circ }}$ longer than the system age, might provide an observational signature of the hot-Jupiter migration process via planet-planet scattering. We 
evaluated $t_{\text {circ }}$ for the eccentric orbits of YBP1194 and YBP1514. Assuming a tidal quality factor $6 \times 10^{4}<Q_{\mathrm{p}}<2 \times 10^{6}$, we calculated $409 \mathrm{Myr}<t_{\text {circ }}<13.6 \mathrm{Gyr}$ for YBP1194 and $220 \mathrm{Myr}<$ $t_{\text {circ }}<6.9$ Gyr for YBP1514 (see Quinn et al. 2013 for details). Given the solar age of M 67 and the wide range of possible $t_{\text {circ }}$, reflecting the choice of the $Q_{\mathrm{p}}$ and the estimation of the planetary radius, no firm conclusion can be drawn for the origin of the eccentric short-period orbits of these stars. Moreover, further investigations and more RV data are needed to better constrain the eccentricities of these objects (see Pont et al. 2011).

The planet around the giant S364 belongs to the lowpopulated region of periods between $\sim 10$ and $\sim 200$ days and is one of the shortest periods found around evolved stars.

When we examine the current distribution of the Jupitermass planets for RV surveys around FGK stars we find an exoplanet host-rate higher than $10 \%$ for planets with a period of up to a few years and $1.20 \pm 0.38 \%$ at solar metallicity, for very close-in hot-Jupiters with a period shorter than ten days (Cumming et al. 2008; Mayor et al. 2011; Wright et al. 2012). This rate around field stars has been in contrast to the lack of detected planets in both open and globular cluster for several years. Before 2012, the detections were limited to a long-period giant planet around one of the Hyades clump giants (Sato et al. 2007) and to a substellar-mass object in NGC2423 (Lovis \& Mayor 2007). No evidence of short-period giant planets has been presented in the study of Paulson et al. (2004) around main-sequence stars of the Hyades, or in several transit campaigns (Bramich et al. 2005; Mochejska et al. 2005, 2006; Pepper et al. 2008; Hartman et al. 2009). These triggered the hypothesis that the frequency of planet-hosting stars in clusters is lower than in the field. To explain the dichotomy between field and cluster stars, it has been suggested that the cluster environment might have a significant impact on the disk-mass distribution. Eisner et al. (2008), studying disks around stars in the Orion Nebula Cluster (ONC), proposed that most of these stars do not posses sufficient mass in the disk to form Jupiter-mass planets or to support an eventual inward migration. Other scenarios may be attributed to post-formation dynamics, in particular to the influence of close stellar encounters (Spurzem et al. 2009; Bonnell et al. 2001) or to tidal evolution of the hot-Jupiters (Debes \& Jackson 2010) in the dense cluster environment. van Saders \& Gaudi (2011), in contrast, found no evidence in support of a fundamental difference in the short-period planet population between clusters and field stars, and attributed the nondetection of planets in transit surveys to the inadequate number of stars surveyed. This seems to be confirmed by the recent results. Indeed, we can list the discovery of two hot-Jupiters in the Praesepe OC in 2012 (Quinn et al. 2012) and of two sub-Neptune planets in the cluster NGC 6811 as part of The Kepler Cluster Study (Meibom et al. 2013), the new announcement of a hot-Jupiter in the Hyades (Quinn et al. 2013) and now the detection in M67 of three Jupiter-mass planets presented in this work. Quinn et al. (2012) obtained a lower limit on the hot-Jupiter frequency in Praesepe of $3.8_{-2.4}^{+5.0} \%$, which is consistent with that of field stars considering the enriched metallicity of this cluster. Meibom et al. (2013) have found the same properties and frequency of low-mass planets in OCs as around field stars. In our case, for short-period giant planets we derived a frequency of $2_{-1.5}^{+3.0} \%$ (errors computed according to Gehrels 1986); which is slightly higher than the value for field stars. Adding giant planets with long periods, the rate becomes $3.4_{-1.7}^{+3.3} \%$, but this fraction is a lower limit that will increase with the follow-up of some other candidates (see Pasquini et al. 2012), which reveal suggestive signals for additional planetary companions. If these were confirmed, the frequency of giant planets would rise to $13_{-2.5}^{+5.0} \%$, in agreement with the rate of giant planets found by Mayor et al. (2011) for field stars.

Acknowledgements. L.P. acknowledges the Visiting Researcher program of the CNPq Brazilian Agency, at the Fed. Univ. of Rio Grande do Norte, Brazil. RPS thanks ESO DGDF, the Hobby Eberly Telescope (HET) project, the PNPS and PNP of INSU - CNRS for allocating the observations. M.T.R. received support from PFB06 CATA (CONICYT). We are grateful to Gaspare Lo Curto and Michele Cappetta for the support in data reduction analysis and helpful discussions.

\section{References}

Baranne, A., Queloz, D., Mayor, M., et al. 1996, A\&AS, 119, 373 Bonnell, I. A., Smith, K. W., et al. 2001, MNRAS, 322, 859

Bouchy, F., \& SOPHIE Team 2006, in Tenth Anniversary of 51 Peg-b, eds.

L. Arnold, F. Bouchy, \& C. Moutou (Paris: Frontier Group), 319 Bramich, D. M., Horne, K., Bond, I. A., et al. 2005, MNRAS, 359, 1096 Cappetta, M., Saglia, R. P., Birkby, J. L., et al. 2012, MNRAS, 427, 1877 Cumming, A., Butler, R. P., Marcy, G. W., et al. 2008, PASP, 120, 531 Debes, J. H., \& Jackson, B. 2010, ApJ, 723, 1703

Eisner, J. A., Plambeck, R. L., Carpenter, J. M., et al. 2008, ApJ, 683, 304 Gehrels, N. 1986, ApJ, 303, 336

Girardi, L., Bressan, A., Bertelli, G., \& Chiosi, C. 2000, A\&AS, 141, 371

Hartman, J. D., Gaudi, B. S., Holman, M. J., et al. 2009, ApJ, 695, 336

Horne, J. H., \& Baliunas, S. L. 1986, ApJ, 302, 757

Lee, Y. S., Beers, T. C., Sivarani, T., et al. 2008, AJ, 136, 2050

Lovis, C., \& Mayor, M. 2007, A\&A, 472, 657

Mathieu, R. D., Latham, D. W., Griffin, R. F., \& Gunn, J. E. 1986, AJ, 92, 1100

Mayor, M., Marmier, M., Lovis, C., et al. 2011, A\&A, submitted [arXiv: 1109.2497]

Mayor, M., Pepe, F., Queloz, D., et al. 2003, The Messenger, 114, 20

Meibom, S., Torres, G., Fressin, F., et al. 2013, Nature, 499, 55

Mermilliod, J.-C., \& Mayor, M. 2007, A\&A, 470, 919

Mochejska, B. J., Stanek, K. Z., Sasselov, D. D., et al. 2005, AJ, 129, 2856 Mochejska, B. J., Stanek, K. Z., Sasselov, D. D., et al. 2006, AJ, 131, 1090 Montgomery, K. A., Marschall, L. A., \& Janes, K. A. 1993, AJ, 106, 181

Önehag, A., Korn, A., Gustafsson, B., et al. 2011, A\&A, 528, A85

Pace, G., \& Pasquini, L. 2004, A\&A, 426, 1021

Pasquini, L., \& Pallavicini, R. 1991, A\&A, 251, 199

Pasquini, L., Biazzo, K., Bonifacio, P., et al. 2008, A\&A, 489, 677

Pasquini, L., Brucalassi, A., Ruiz, M. T., et al. 2012, A\&A, 545, A139

Paulson, D. B., Saar, S. H., Cochran, W. D., \& Henry, G. W. 2004, AJ, 127, 1644

Pepe, F., Mayor, M., Galland, F., et al. 2002, A\&A, 388, 632

Pepper, J., Stanek, K. Z., Pogge, R. W., et al. 2008, AJ, 135, 907

Perruchot, S., Bouchy, F., et al. 2011, in SPIE Conf. Ser., 8151

Pietrinferni, A., Cassisi, S., Salaris, M., \& Castelli, F. 2004, ApJ, 612, 168

Pont, F., Husnoo, N., Mazeh, T., \& Fabrycky, D. 2011, MNRAS, 414, 1278

Queloz, D., Henry, G. W., Sivan, J. P., et al. 2001, A\&A, 379, 279

Quinn, S. N., White, R. J., Latham, D. W., et al. 2012, ApJ, 756, L33

Quinn, S. N., White, R. J., Latham, D. W., et al. 2013, ApJ, submitted [arXiv: 1310.7328$]$

Randich, S., Bragaglia, A., Pastori, L., et al. 2005, The Messenger, 121, 18

Randich, S., Sestito, P., Primas, F., et al. 2006, A\&A, 450, 557

Sanders, W. L. 1977, A\&AS, 27, 89

Santerne, A., Díaz, R. F., Moutou, C., et al. 2012, A\&A, 545, A76

Sato, B., Izumiura, H., Toyota, E., et al. 2007, ApJ, 661, 527

Scargle, J. D. 1982, ApJ, 263, 835

Smolinski, J. P., Lee, Y. S., Beers, T. C., et al. 2011, AJ, 141, 89

Spurzem, R., Giersz, M., Heggie, D. C., \& Lin, D. N. C. 2009, ApJ, 697, 458

Taylor, B. J. 2007, AJ, 133, 370

Tull, R. G. 1998, in SPIE Conf. Ser. 3355, ed. S. D’Odorico, 387

van den Berg, M., Tagliaferri, G., et al. 2004, A\&A, 418, 509

van Saders, J. L., \& Gaudi, B. S. 2011, ApJ, 729, 63

Wright, J. T., \& Howard, A. W. 2009, ApJS, 182, 205

Wright, J. T., Marcy, G. W., Howard, A. W., et al. 2012, ApJ, 753, 160

Wu, Y., Luo, A.-L., Li, H.-N., et al. 2011, Res. Astron. Astrophys., 11, 924

Yadav, R. K. S., Bedin, L. R., Piotto, G., et al. 2008, A\&A, 484, 609 


\section{Appendix A: CMD and membership probabilities}

In this section we summarize the results presented in Pasquini et al. (2012), focusing in particular on the three stars discussed in the Letter.

YBP1194, YBP1514, and S364 belong to the M 67 sample that includes a total of 88 stars. All targets have $V$ mag between 9 and 15 , and a mass range between $0.9-1.4 M_{\odot}$.

We selected main-sequence stars (included YBP1194 and YBP1514) with a membership probability higher than $60 \%$ and a proper motion shorter than 6 mas/yr with respect to the average according to Yadav et al. (2008). For the giants we refer to Sanders (1977). The RV membership was established for the latter following the work of Mermilliod \& Mayor (2007), who studied the membership and binarity of 123 red giants in six old OCs, and of Mathieu et al. (1986), who made a very complete RV survey of the evolved stars of M67 with a precision of a few hundreds of $\mathrm{m} \mathrm{s}^{-1}$. The majority of the other stars were selected according to Pasquini et al. (2008), who used several VLT-FLAMES exposures for each star to classify suspected binaries. We found that YBP1194, YBP1514, and S364 are probable RV members with a mean RV within onesigma from the average cluster RV. For the latter, we adopted the value of $\left\langle\mathrm{RV}_{\mathrm{M} 67}\right\rangle=33.724 \mathrm{~km} \mathrm{~s}^{-1}$ and the dispersion of $\sigma= \pm 0.646 \mathrm{~km} \mathrm{~s}^{-1}$ estimated in Pasquini et al. (2012).

Table 3 shows proper motions and membership probability for the three stars discussed. Details about selection criteria and motion errors can be found in the original Yadav et al. (2008) and Sanders (1977) works.

In Fig. A.1, we report the observed region of the colormagnitude diagram (CMD), indicating in different colors the the position of the stars considered in this letter and the solar ana$\log$, as determined in Pasquini et al. (2008). The three stars analyzed in this work lie quite well on the cluster sequence in the CMD. We superimposed the isochrones from Pietrinferni et al. (2004) with solar metallicity and age corresponding to $3.5 \mathrm{Gyr}$ (black curve), 4.0 Gyr (dark-blue curve) and 4.5 Gyr (light-blue curve). We also included the $4.0 \mathrm{Gyr}$ isochrone (red curve) with a slightly lower reddening $(E(B-V)=0.02$ instead of 0.041 , Taylor 2007). This curve seems to match the colors of the turnoff better (see also the discussion in Pasquini et al. 2012). In the same figure, we report the Padova isochrone using $E(B-V)=$ $0.041 \pm 0.004$, with solar metallicity, age $4.47 \mathrm{Gyr}$, and $Y=0.26$ (Girardi et al. 2000).

Given that the values of stellar parameters have influence on the estimation of the planet masses, we evaluated the effects on the host star masses and radii of using isochrones with different ages and slightly lower reddening. While for the two

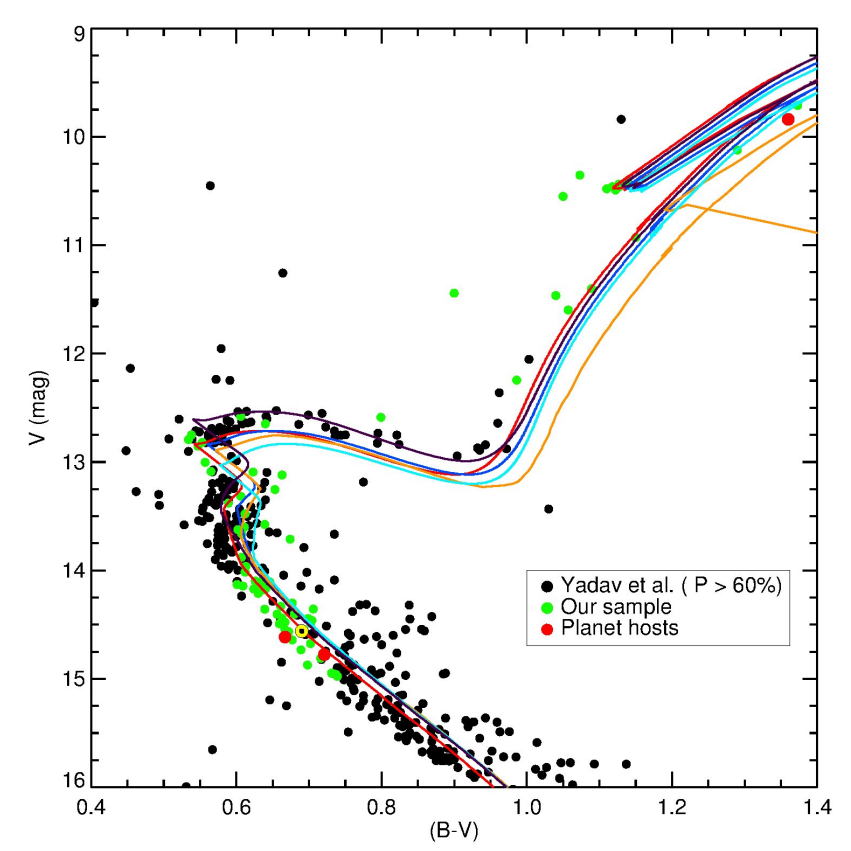

Fig. A.1. CMD of M 67 (photometry from Yadav et al. 2008) for probable members $\left(P_{\mu}>60 \%\right)$. The isochrones are taken from the BaSTI library (Pietrinferni et al. 2004). The isochrones in black, dark blue, and light blue correspond to $3.5 \mathrm{Gyr}$, 4.0 Gyr, and $4.5 \mathrm{Gyr}$ with a reddening $E(B-V)=0.041 \pm 0.004$ (Taylor 2007). The isochrone in red is a $4.0 \mathrm{Gyr}$ with a lower reddening $(E(B-V)=0.02)$. The isochrone in orange is a $4.47 \mathrm{Gyr}$ from Girardi et al. (2000) with $E(B-V)=0.041 \pm 0.004$. The location of the Sun, if it were within M 67, is marked with a $\odot$ in yellow.

Table A.1. Object ID, proper motions, and membership probability of the targets; reference.

\begin{tabular}{lcccc}
\hline \hline Object & $\mu_{\mathrm{x}} \pm \Delta \mu_{\mathrm{x}}$ & $\mu_{\mathrm{y}} \pm \Delta \mu_{\mathrm{y}}$ & Prob\% & Reference \\
\hline YBP1194 & $0.30 \pm 1.01$ & $-0.42 \pm 0.65$ & 99 & Yadav et al. (2008) \\
YBP1514 & $-0.12 \pm 1.13$ & $1.73 \pm 1.37$ & 98 & Yadav et al. (2008) \\
S364 & -0.088 & 0.164 & 82 & Sanders (1977) \\
\hline
\end{tabular}

main-sequence stars YBP1194 and YBP1514 we found no significant incidence, for the giant S364, an age uncertainty of $\pm 0.5 \mathrm{Gyr}$ and a lower reddening would induce an error on the star mass of $4 \%$ and on its radii of $3 \%$. Therefore, we decided to include this effect in the uncertainties of S364 listed in Table 1 and in the error of the planet mass. 
Table A.2. Relative RV measurements, RV uncertainties, bisector span, and ratio of the $\mathrm{H}_{\alpha}$ core with respect to the continuum (see Pasquini \& Pallavicini 1991) for YBP1194.

\begin{tabular}{lrrrrl}
\hline \hline $\begin{array}{l}\text { BJD } \\
(-2450000)\end{array}$ & $\begin{array}{r}\mathrm{RV} \\
\left(\mathrm{km} \mathrm{s}^{-1}\right)\end{array}$ & $\begin{array}{r}\sigma \mathrm{RV} \\
\left(\mathrm{km} \mathrm{s}^{-1}\right)\end{array}$ & $\begin{array}{r}\text { BIS span } \\
\left(\mathrm{km} \mathrm{s}^{-1}\right)\end{array}$ & $\mathrm{H}_{\alpha}$ ratio & Instrument \\
\hline 4489.51193 & -0.000 & 0.009 & -0.021029 & 0.038294 & Sophie \\
4491.50617 & -0.030 & 0.009 & -0.042000 & 0.038689 & Sophie \\
4842.84025 & 0.051 & 0.010 & -0.018851 & 0.038897 & HARPS \\
4856.62544 & 0.013 & 0.012 & -0.081895 & 0.038995 & HARPS \\
4862.59495 & 0.031 & 0.013 & -0.009653 & 0.039465 & HARPS \\
5188.83049 & 0.002 & 0.010 & -0.056037 & 0.039817 & HARPS \\
5189.82037 & 0.010 & 0.010 & 0.022874 & 0.039387 & HARPS \\
5190.79901 & 0.036 & 0.009 & 0.039774 & 0.038994 & HARPS \\
5214.85851 & -0.030 & 0.013 & 0.008036 & 0.039872 & HARPS \\
5216.70466 & -0.000 & 0.012 & 0.051777 & 0.037107 & HARPS \\
5594.79168 & 0.019 & 0.014 & -0.021881 & 0.039218 & HARPS \\
5977.66236 & 0.037 & 0.010 & -0.028000 & 0.037126 & HARPS \\
5986.51471 & -0.034 & 0.009 & -0.057833 & 0.038907 & Sophie \\
6219.98852 & 0.049 & 0.027 & -0.025204 & 0.037364 & HET \\
6243.93406 & -0.028 & 0.028 & 0.019138 & 0.036729 & HET \\
6245.81040 & -0.007 & 0.017 & -0.026488 & 0.041533 & HARPS \\
6270.77262 & 0.009 & 0.019 & -0.053592 & 0.039405 & HARPS \\
6286.00446 & -0.066 & 0.025 & -0.042748 & 0.036126 & HET \\
6305.17913 & 0.017 & 0.014 & -0.092977 & 0.038956 & HARPS \\
6316.76841 & 0.005 & 0.012 & -0.021029 & 0.039405 & HARPS \\
6322.71086 & -0.006 & 0.021 & -0.028423 & 0.038896 & HARPS \\
6338.66079 & 0.034 & 0.026 & -0.095893 & 0.037364 & HET \\
6378.72343 & 0.002 & 0.026 & 0.024230 & 0.038289 & HET \\
\hline
\end{tabular}

Notes. All the RV data points are corrected to the zero point of HARPS.

Table A.3. Relative RV measurements, RV uncertainties, bisector span, and ratio of the $\mathrm{H}_{\alpha}$ core with respect to the continuum (see Pasquini \& Pallavicini 1991) for YBP1514.

\begin{tabular}{lrrrrl}
\hline \hline $\begin{array}{l}\text { BJD } \\
(-2450000)\end{array}$ & $\begin{array}{r}\mathrm{RV} \\
\left(\mathrm{km} \mathrm{s}^{-1}\right)\end{array}$ & $\begin{array}{r}\sigma \mathrm{RV} \\
\left(\mathrm{km} \mathrm{s}^{-1}\right)\end{array}$ & $\begin{array}{r}\text { BIS span } \\
\left(\mathrm{km} \mathrm{s}^{-1}\right)\end{array}$ & $\mathrm{H}_{\alpha}$ ratio & Instrument \\
\hline 4858.72562 & -0.037 & 0.017 & -0.097357 & 0.040203 & HARPS \\
4861.71515 & 0.008 & 0.012 & 0.030042 & 0.039426 & HARPS \\
5214.87795 & 0.008 & 0.020 & -0.056154 & 0.037161 & HARPS \\
5216.72426 & -0.047 & 0.014 & 0.015647 & 0.042089 & HARPS \\
5260.70288 & 0.003 & 0.013 & -0.042552 & 0.039905 & HARPS \\
5269.72337 & 0.059 & 0.014 & 0.035491 & 0.038077 & HARPS \\
5595.74177 & -0.029 & 0.014 & 0.026610 & 0.039282 & HARPS \\
5626.15072 & -0.039 & 0.014 & -0.057171 & 0.039905 & HARPS \\
5943.29835 & -0.037 & 0.013 & 0.015647 & 0.040103 & HARPS \\
5967.45833 & 0.008 & 0.023 & -0.084845 & 0.039356 & HARPS \\
5968.59423 & -0.031 & 0.014 & -0.020833 & 0.039036 & HARPS \\
5977.68405 & 0.007 & 0.012 & -0.051313 & 0.039475 & HARPS \\
5986.56139 & 0.068 & 0.010 & -0.020833 & 0.039306 & Sophie \\
6036.66109 & -0.005 & 0.025 & -0.054711 & 0.040201 & HET \\
6245.83389 & -0.037 & 0.015 & 0.025824 & 0.040109 & HARPS \\
6254.90496 & -0.036 & 0.026 & -0.035278 & 0.040001 & HET \\
6270.81399 & -0.068 & 0.019 & 0.014902 & 0.039345 & HARPS \\
6305.22499 & -0.022 & 0.016 & -0.031263 & 0.037032 & HARPS \\
6307.81028 & -0.036 & 0.016 & -0.016596 & 0.039764 & HARPS \\
6317.74917 & -0.049 & 0.014 & -0.071034 & 0.038687 & HARPS \\
6322.68946 & 0.016 & 0.019 & -0.084845 & 0.040020 & HARPS \\
6332.68338 & -0.021 & 0.016 & -0.012640 & 0.039854 & HARPS \\
6335.68811 & 0.001 & 0.025 & 0.056131 & 0.041020 & HET \\
6339.68307 & 0.066 & 0.025 & -0.056093 & 0.042437 & HET \\
6364.59175 & 0.007 & 0.024 & 0.011085 & 0.040285 & HET \\
\hline & & & & &
\end{tabular}

Notes. All the RV data points are corrected to the zero point of HARPS. 
A. Brucalassi et al.: Three planetary companions around M 67 stars

Table A.4. Relative RV measurements, RV uncertainties, bisector span, and ratio of the $\mathrm{H}_{\alpha}$ core with respect to the continuum (see Pasquini \& Pallavicini 1991) for S364.

\begin{tabular}{|c|c|c|c|c|c|}
\hline $\begin{array}{l}\text { BJD } \\
(-2450000)\end{array}$ & $\begin{array}{r}\mathrm{RV} \\
\left(\mathrm{km} \mathrm{s}^{-1}\right) \\
\end{array}$ & $\begin{array}{r}\sigma \mathrm{RV} \\
\left(\mathrm{km} \mathrm{s}^{-1}\right) \\
\end{array}$ & $\begin{array}{r}\text { BIS span } \\
\left(\mathrm{km} \mathrm{s}^{-1}\right)\end{array}$ & $\mathrm{H}_{\alpha}$ ratio & Instrument \\
\hline 2647.77191 & -0.030 & 0.010 & 0.035310 & - & Coralie \\
\hline 2682.68790 & -0.070 & 0.010 & -0.004670 & - & Coralie \\
\hline 2695.70992 & -0.077 & 0.011 & 0.022800 & - & Coralie \\
\hline 3004.82521 & 0.009 & 0.012 & 0.046980 & - & Coralie \\
\hline 3020.78490 & -0.004 & 0.021 & 0.042690 & - & Coralie \\
\hline 3046.73656 & -0.016 & 0.010 & -0.021670 & - & Coralie \\
\hline 3055.71453 & -0.039 & 0.012 & 0.010630 & - & Coralie \\
\hline 4855.58011 & -0.037 & 0.002 & 0.042682 & 0.038161 & HARPS \\
\hline 4860.32500 & -0.036 & 0.008 & 0.042690 & 0.038048 & Sophie \\
\hline 5216.82809 & -0.012 & 0.002 & 0.071695 & 0.038278 & HARPS \\
\hline 5594.58582 & -0.032 & 0.003 & 0.071762 & 0.038105 & HARPS \\
\hline 5977.55818 & -0.071 & 0.002 & 0.064832 & 0.037733 & HARPS \\
\hline 5985.30291 & -0.059 & 0.007 & 0.022666 & 0.038426 & Sophie \\
\hline 6236.94284 & 0.004 & 0.018 & 0.050689 & 0.040027 & HET \\
\hline 6245.76368 & 0.034 & 0.003 & 0.059181 & 0.038501 & HARPS \\
\hline 6245.86577 & 0.041 & 0.002 & 0.052050 & 0.038271 & HARPS \\
\hline 6269.71823 & 0.046 & 0.003 & 0.061007 & 0.041626 & HARPS \\
\hline 6270.75931 & 0.063 & 0.003 & 0.059963 & 0.038616 & HARPS \\
\hline 6302.68302 & -0.006 & 0.003 & 0.057069 & 0.038056 & HARPS \\
\hline 6305.24544 & -0.018 & 0.002 & 0.036772 & 0.037843 & HARPS \\
\hline 6309.75038 & -0.010 & 0.002 & 0.056751 & 0.039626 & HARPS \\
\hline 6309.75141 & -0.042 & 0.018 & 0.042921 & 0.039879 & HET \\
\hline 6324.67965 & -0.056 & 0.004 & 0.045385 & 0.038311 & HARPS \\
\hline 6331.69998 & -0.051 & 0.020 & 0.043008 & 0.039356 & HET \\
\hline 6332.69784 & -0.038 & 0.003 & 0.045065 & 0.038067 & HARPS \\
\hline 6345.65885 & -0.072 & 0.020 & 0.041555 & 0.039587 & HET \\
\hline 6376.73341 & 0.072 & 0.021 & 0.022076 & 0.039445 & HET \\
\hline
\end{tabular}

Notes. All the RV data points are corrected to the zero point of HARPS. 